\title{
Size Scaling of Plastic
} Deformation in Simple Shear: Fractional Strain-Gradient

Carl F. 0. Dahlberg ${ }^{1}$ Senior Research Associate
artment of Solid Mechanics, Royal Institute of Technology (KTH), Stockholm 10044, Sweden e-mail: carldah|@kth.se

Michael Ortiz Mem. ASME Professor Division of Engineering and Applied Science, California Institute of Technology, Pasadena, CA 91125 e-mail: ortiz@aero.caltech.edu

\section{Plasticity and Boundary Effects in Conventional Strain-Gradient Plasticity}

A recently developed model based on fractional derivatives of plastic strain is compared with conventional strain-gradient plasticity (SGP) models. Specifically, the experimental data and observed model discrepancies in the study by Mu et al. (2016, "Dependence of Confined Plastic Flow of Polycrystalline Cu Thin Films on Microstructure," MRS Com. Res. Let. 20, pp. 1-6) are considered by solving the constrained simple shear problem. Solutions are presented both for a conventional SGP model and a model extension introducing an energetic interface. The interface allows us to relax the Dirichlet boundary condition usually assumed to prevail when solving this problem with the SGP model. We show that the particular form of a relaxed boundary condition does not change the underlying size scaling of the yield stress and consequently does not resolve the scaling issue. Furthermore, we show that the fractional strain-gradient plasticity model predicts a yield stress with a scaling exponent that is equal to the fractional order of differentiation.

[DOI: 10.1115/1.4045872]

Keywords: constitutive modeling of materials, mechanical properties of materials, plasticity

\section{Introduction}

This paper investigates the ability of two classes of nonlocal plasticity theories to reproduce experimentally observed size scalings of flow stress in small-scale metal specimens. The work is motivated, in part, by the size-dependent yielding observed in the recent experiments of constrained shearing of thin copper films by $\mathrm{Mu}$ et al. [1-3]. Their results, spanning about a decade in film thickness, show a yield stress inversely proportional to thickness raised to the power of $\sim 0.2$. As noted in Ref. [2], this moderate size scaling cannot be reproduced by conventional strain-gradient plasticity (SGP) theories where the size dependence is much stronger, namely, inversely proportional to the size of the specimen.

Higher order strain-gradient plasticity (henceforth termed conventional SGP) has been developed in response to extensive experimental evidence of size-dependent plastic deformation of metallic specimens at small spatial scales. The first such results are often attributed to Hall [4] and Petch [5], which resulted in the celebrated Hall-Petch equation where the yield point of a polycrystal depends on the inverse of the square root of the grain diameter. Since classical local continuum theories of plasticity are scale invariant (e.g., $J_{2}$ flow theory), this class of theories cannot predict a sizedependent response. On the other hand, SGP theories, see, e.g., Refs. [6-9], introduces a size scaling in the continuum by including a dependence on plastic strain gradients and a material length scale parameter, usually denoted as $\ell$, in the internal work. This extension makes the continuum nonlocal and changes the structure of the

\footnotetext{
${ }^{1}$ Corresponding author.

Contributed by the Applied Mechanics Division of ASME for publication in the Journal of ApPLIED Mechanics. Manuscript received November 4, 2019; final manuscript received December 18, 2019; published online January 3, 2020. Assoc. Editor: Alan Needleman.
}

boundary value problem such that the so-called higher order boundary conditions need to be prescribed in addition to the standard traction/displacement conditions. When solving a SGP boundary value problem, that contains strain gradients imposed either by the loading and/or geometry or by prescribing constraints on plastic strain through the higher order boundary conditions, the resulting stress-strain response exhibits an intrinsic size dependence. The issue at hand here is how this size dependence manifests itself and how it compares with the experimental data.

As noted already by Evans and Hutchinson [10], conventional SGP significantly overestimates the thickness-dependent increase in the bending moment at yielding when fitting with the data from bending of thin foils $[11,12]$. With the recent experimental protocol developed in Refs. $[1,2]$, where a thin layer of copper is sandwiched between thick layers of stiff and elastic ceramics, it is now also possible to get detailed experimental data on the "canonical problem posed and analyzed in most papers on SGP formulations" [2], namely, a constrained simple shear configuration where a strip of ductile material is plastically confined $\left(\varepsilon^{\mathrm{p}}=0\right.$ at the boundaries) between two rigid blocks and then sheared. The problem is illustrated in Fig. 1. When this problem is solved within a SGP framework, the resulting shear stress at yielding, $\tau_{\mathrm{y}}$, is strongly dependent on the ratio $\ell / h$ of the material length scale to the thickness of the strip. The scaling predicted from SGP models is almost always reported as $\tau_{\mathrm{y}} \propto(\ell / h)^{\alpha}$, with $\alpha \simeq 1$, whereas the experimental data [1,2] reveal a weaker scaling where $0.2 \leq \alpha \leq 0.7$. A number of extensions of conventional SGP, e.g., by changing the definition of the internal variable in terms of plastic strain and plastic strain gradients [10,13-17] or by modifying the constitutive length scale by having it evolve with deformation [10,18-20], have been proposed. Although these extensions can give quite different material responses and can be made to fit better to experiments, compared with 


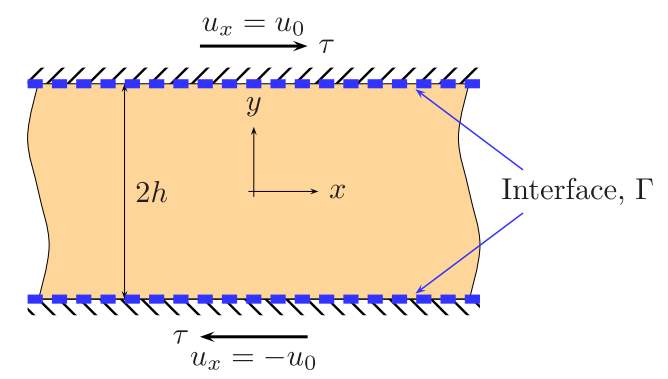

Fig. 1 Simple shear of a layer of thickness $2 h$ sandwiched between two rigid blocks. Interface between the rigid block (boundary condition) and elastic-plastic layer (solution domain) is highlighted.

conventional SGP, currently, there is no indication that they strongly influence the size scaling.

In a recent paper [21], we have proposed a model that accounts for the experimentally observed scaling by relaxing the overly stiff differential structure of strain-gradient plasticity. This relaxation is accomplished by introducing a dependence of the plastic strain on fractional derivatives in the free energy and referred. We refer to the resulting model as fractional strain-gradient plasticity (FSGP). It should also be noted that fractional derivatives are not entirely unconventional and have successfully been used in mechanics to model, e.g., elasticity [22], viscoelasticity [23], nonassociative plastic flow [24,25], and ductile fracture [26-28]. However, one important issue not addressed in detail in Ref. [21] concerns the appropriate choice of higher order boundary conditions. Thus, to get a size-dependent response in the simple shear configuration, the boundary conditions must, to some extent, constrain development of plastic strain at $y= \pm h$. Typically, in SGP, this constraining effect is accomplished by applying Dirichlet conditions on the plastic shear strain $\left(\gamma^{\mathrm{p}}( \pm h)=0\right)$, which then lead to a nonhomogeneous plastic strain distribution and therefore gradient effects. In the absence of such conditions, the solution reduces to the size-independent local theory with $\tau_{\mathrm{y}}=\tau_{0}$. A prudent question then concerns whether these so-called micro hard boundary conditions are reasonable as a description of the plastic strain state at an interface.

In this paper, we investigate what happens if the state at the boundary is relaxed from an explicit micro hard condition. We do this by introducing an interface at the boundary and model this interface by a free energy contribution. This simple device allows us to effectively model any boundary condition from a micro hard to a micro free and, importantly, any state in between these two. In particular, we are not constrained by the binary choice of prescribing either a Dirichlet or a Neumann condition but can smoothly interpolate between these two conditions. We specifically compare conventional SGP and the influence of higher order boundary conditions with results from fractional straingradient plasticity.

We note that, also motivated by the same set of experiments and scaling discrepancies detailed earlier, Kuroda and Needleman [29] have recently proposed a model that focuses on the higher order boundary conditions. Specifically, they investigate the response of the simple shear problem when the boundary conditions are changed from Dirichlet to a Neumann when a sufficiently large plastic strain gradient is attained at the boundary. Their central result is that, for such variable boundary conditions, the shear stress scales with layer thickness in a more complex manner than just $\alpha=1$. In this manner, the model of Ref. [29] provides an alternative means of closing the gap between conventional SGP and experimental observations. Of course, it is entirely possible that the observed scaling behavior is the result of a combination of mechanisms acting concurrently, including fractional straingradient plasticity, complex higher order boundary conditions, and others as yet to be identified.

\section{Strain-Gradient Plasticity With Energetic Interfaces}

Following Gudmundson [7], the internal virtual work, $\delta W_{i}$, in a volume $V$ is assumed to include contribution from elastic and plastic processes as follows:

$$
\delta W_{\mathrm{i}}=\int_{V}\left[\sigma_{i j} \delta \varepsilon_{i j}^{\mathrm{e}}+q_{i j} \delta \varepsilon_{i j}^{\mathrm{p}}+m_{i j k} \delta \varepsilon_{i j, k}^{\mathrm{p}}\right] \mathrm{d} V
$$

where $\sigma_{i j}$ is the Cauchy stress. Work conjugates to plastic strain and plastic strain gradients are the microstress $q_{i j}$ and momentstress $m_{i j k}$, respectively. An application of Gauss' theorem gives

$$
\begin{aligned}
\delta W_{\mathrm{i}}= & \int_{V}\left[\sigma_{i j} n_{j} \delta u_{j}^{\mathrm{e}}+m_{i j k} n_{k} \delta \varepsilon_{i j}^{\mathrm{p}}\right] \mathrm{d} S \\
& -\int_{V}\left[\sigma_{i j, j} \delta u_{i}+\left(m_{i j k, k}+s_{i j}-q_{i j}\right) \delta \varepsilon_{i j}^{\mathrm{p}}\right] \mathrm{d} V
\end{aligned}
$$

where $S$ is the surface boundary of $V$ with outward normal vector $n_{i}$, and $s_{i j}=\sigma_{i j}-\delta_{i j} \sigma_{k k} / 3$ is the Cauchy stress deviator. The variations of displacements $\delta u_{i}$ and plastic strains $\delta \varepsilon_{i j}^{p}$ should vanish on parts of $S$ where $u_{i}$ or $\varepsilon_{i j}^{\mathrm{p}}$ are prescribed, respectively. Introducing $T_{i}=\sigma_{i j} n_{j}$ as the traction vector and $M_{i j}=m_{i j k} n_{k}$ as the higher order moment traction, the first integral can be identified as the external virtual work $\delta W_{\mathrm{e}}$. The second integral should vanish for arbitrary variations, resulting in the following set of equilibrium equations:

$$
\begin{gathered}
\sigma_{i j, j}=0 \\
m_{i j k, k}+s_{i j}-q_{i j}=0
\end{gathered}
$$

The principle of virtual work can be written as follows:

$$
\begin{aligned}
& \int_{V}\left[\sigma_{i j} \delta \varepsilon_{i j}+\left(q_{i j}-s_{i j}\right) \delta \varepsilon_{i j}^{\mathrm{p}}+m_{i j k} \delta \varepsilon_{i j, k}^{\mathrm{p}}\right] \mathrm{d} V \\
& =\int_{S}\left[T_{i} \delta u_{i}+M_{i j} \delta \varepsilon_{i j}^{\mathrm{p}}\right] \mathrm{d} S
\end{aligned}
$$

When internal interfaces are considered, a contribution to the internal work appears on the left hand side as follows:

$$
\delta W_{\Gamma}=\int_{\Gamma}\left[T_{i}^{I} \delta u_{i}^{I}+M_{i j}^{I} \delta \varepsilon_{i j}^{\mathrm{p} I}\right] \mathrm{d} \Gamma
$$

where $I=1,2$ denotes either side of the interface.

2.1 Constitutive Relations. In light of the enhanced continuum description in Eq. (1), it is natural to introduce a free energy per unit volume $\psi$ that in the most general case depends on $\varepsilon_{i j}^{\mathrm{e}}, \varepsilon_{i j}^{\mathrm{p}}$, and $\varepsilon_{i j, k}^{\mathrm{p}}$. The rate of dissipation may then be expressed as the difference between the internal energy work rate and the rate of change of free energy

$$
\int_{V}\left[\left(\sigma_{i j}-\frac{\partial \psi}{\partial \varepsilon_{i j}^{\mathrm{e}}}\right) \dot{\varepsilon}_{i j}^{\mathrm{e}}+\left(q_{i j}-\frac{\partial \psi}{\partial \varepsilon_{i j}^{\mathrm{p}}}\right) \dot{\varepsilon}_{i j}^{\mathrm{p}}+\left(m_{i j k}-\frac{\partial \psi}{\partial \varepsilon_{i j, k}^{\mathrm{p}}}\right) \dot{\varepsilon}_{i j, k}^{\mathrm{p}}\right] \mathrm{d} V \geq 0
$$

The dissipation must be nonnegative in every point in the body, which can be expressed as follows:

$$
\bar{\sigma}_{i j} \dot{\varepsilon}_{i j}^{\mathrm{e}}+\bar{q}_{i j} \dot{\varepsilon}_{i j}^{\mathrm{p}}+\bar{m}_{i j k} \dot{\varepsilon}_{i j, k}^{\mathrm{p}} \geq 0
$$

where

$$
\bar{\sigma}_{i j}=\sigma_{i j}-\frac{\partial \psi}{\partial \varepsilon_{i j}^{\mathrm{e}}}, \quad \bar{q}_{i j}=q_{i j}-\frac{\partial \psi}{\partial \varepsilon_{i j}^{\mathrm{p}}}, \quad \bar{m}_{i j k}=m_{i j k}-\frac{\partial \psi}{\partial \varepsilon_{i j, k}^{\mathrm{p}}}
$$

In accordance with standard elasticity, the Cauchy stress is $\sigma_{i j}=C_{i j k l} \varepsilon_{k l}^{\mathrm{e}}$, where $C_{i j k l}$ is the standard linear elasticity tensor, and hence, $\bar{\sigma}_{i j}=0$. It is assumed that the nonstandard stresses 
contribute only to dissipate work such that $\bar{q}_{i j}=q_{i j}$ and $\bar{m}_{i j k}=m_{i j k}$, and the dissipation inequality is then

$$
q_{i j} \dot{\varepsilon}_{i j}^{\mathrm{p}}+m_{i j k} \dot{\varepsilon}_{i j, k}^{\mathrm{p}} \geq 0
$$

Constitutive relations for $q_{i j}$ and $m_{i j k}$ can be formulated such that the stresses are always collinear with the corresponding conjugated rates, which ensure that Eq. (10) is always fulfilled. A general ratedependent case may be constructed as a pair of flow rules:

$$
\begin{aligned}
\dot{\varepsilon}_{i j}^{\mathrm{p}} & =g\left(\sigma_{\mathrm{f}}, \Sigma\right) q_{i j} \\
\dot{\varepsilon}_{i j, k}^{\mathrm{p}} & =g\left(\sigma_{\mathrm{f}}, \Sigma\right) \frac{m_{i j k}}{\ell^{2}}
\end{aligned}
$$

where $g$ is a function of the flow stress $\sigma_{\mathrm{f}}$ and an effective stress measure $\Sigma$, and $\ell$ is a constitutive length scale parameter. The flow stress can be a function of the equivalent plastic strain $E^{\mathrm{p}}$ as follows:

$$
\sigma_{\mathrm{f}}=\sigma_{0}+f_{\mathrm{h}}\left(E^{\mathrm{p}}\right)
$$

where $\sigma_{0}$ is the large-scale initial yield stress in uniaxial tension and $f_{\mathrm{h}}$ is some hardening function that depends on $E^{\mathrm{p}}$.

In the absence of plastic strain gradient the model should reduce to standard plasticity theory. Effective quantities are therefore calculated as follows:

$$
\dot{E}^{\mathrm{p}}=\sqrt{\frac{2}{3}\left(\dot{\varepsilon}_{i j}^{\mathrm{p}} \dot{\varepsilon}_{i j}^{\mathrm{p}}+\ell^{2} \dot{\varepsilon}_{i j, k}^{\mathrm{p}} \dot{\varepsilon}_{i j, k}^{\mathrm{p}}\right)}, \quad E^{\mathrm{p}}=\int_{0}^{t} \dot{E}^{\mathrm{p}} \mathrm{d} t
$$

and

$$
\Sigma=\sqrt{\frac{3}{2}\left(q_{i j} q_{i j}+\frac{m_{i j k} m_{i j k}}{\ell^{2}}\right)}
$$

that in the large-scale limit reduces to standard $J_{2}$ plasticity. Finally, the function $g$ is specified as follows:

$$
g\left(\sigma_{\mathrm{f}}, \Sigma\right)=\frac{3 \dot{\varepsilon}_{0}}{2 \sigma_{\mathrm{f}}}\left[\eta+\left(\frac{\Sigma}{\sigma_{\mathrm{f}}}\right)^{n}\right]
$$

where the strain rate sensitivity exponent $n \gg 1$ has been introduced to mimic rate-independent plasticity and $\eta \ll 1$ is a parameter that is necessary from a numerical point of view. This closes the constitutive description of the bulk material.

2.1.1 Energetic Interface. Internal interfaces require additional consideration. The interface is assumed to have vanishing thickness in relation to $h$ and $\ell$ and can therefore be modeled as the surface separating material 1 and 2 . Here, material 1 denotes the elasticplastic strip under consideration and material 2 denotes the purely elastic material on either side of the strip to be excluded from the analysis. First, we assume that the interface does not slide or separate implying that displacements are continuous $u_{i}^{(1)}=u_{i}^{(2)}$. In relation to displacements and force tractions, the interface does not expend any work and goes undetected.

The plastic processes across the interface must satisfy thermodynamic restrictions and we formulate the rate of dissipation $\dot{\Theta}_{\Gamma}$ as the difference between the work rate and the rate of change of internal energy

$$
\dot{\Theta}_{\Gamma}=\left(M_{i j}-\frac{\partial W_{\Gamma}}{\partial \varepsilon_{i j}^{\mathrm{p}}}\right) \dot{\varepsilon}_{i j}^{\mathrm{p}} \geq 0
$$

We restrict plastic dissipation to processes in the bulk and assume that $\dot{\Theta}_{\Gamma}=0$, which implies

$$
M_{i j}=\frac{\partial W_{\Gamma}}{\partial \varepsilon_{i j}^{\mathrm{p}}}
$$

Thus, the state at the interface is governed by the strain energy $W_{\Gamma}\left(\varepsilon_{i j}^{\mathrm{p}(1)}, \varepsilon_{i j}^{\mathrm{p}(2)}\right)$, where $\varepsilon_{i j}^{\mathrm{p}(1)}, \varepsilon_{i j}^{\mathrm{p}(2)}$ denotes the plastic strain state at either side of $\Gamma$.
Different functional forms for this energy have been investigated by, e.g., Refs. $[8,9,17,30]$. Here, we restrict attention to the case where $\varepsilon_{i j}^{p(2)}=0$, since only the material on one side of the interface experiences plastic straining. Furthermore, it is sufficient to consider a quadratic form in plastic strains (see, e.g., results in Refs. $[17,31])$ such that the moment tractions are a linear function of $\varepsilon_{i j}^{\mathrm{p} 1}$,

$$
M_{i j}=\kappa_{\Gamma} \varepsilon_{i j}^{\mathrm{p}(1)}
$$

where $\kappa_{\Gamma}$ is an interface modulus. This simple formulation still has the attractive property that it interpolates between two extreme behaviors with respect to the development of plastic strains at the interface. If $\kappa_{\Gamma} \rightarrow \infty$, the interface penalizes plastic strains and behaves as a so-called micro hard boundary condition $\left(\varepsilon_{i j}^{\mathrm{p}(1)}=0\right)$, and conversely, if $\kappa_{\Gamma} \rightarrow 0$, the interface does not influence the plastic strains and behaves as the so-called micro free condition $\left(M_{i j}^{(1)}=0\right)$. It should be noted that this formulation allow discontinuities in plastic strain across the interface.

\section{Fractional Strain-Gradient Plasticity}

In a recent paper, the authors introduced a theory for FSGP. The motivation for developing FSGP was presented in the results in Refs. [1-3] in relation to the simple shear problem and the scaling issues discussed earlier. The details can be found in Ref. [21] but are briefly summarized for completeness.

3.1 Gradient Plasticity Preliminaries and Deformation Theory Formulation. In correspondence with Sec. 2.1, it is assumed that a free energy density exists on the form

$$
\psi=\psi\left(\varepsilon_{i j}^{\mathrm{e}}, \varepsilon_{i j}^{\mathrm{p}}, \varepsilon_{i j, k}^{\mathrm{p}},\right)=\psi_{\mathrm{e}}\left(\varepsilon_{i j}^{\mathrm{e}}\right)+\psi_{\mathrm{p}}\left(\varepsilon_{i j}^{\mathrm{p}}\right)+\psi_{\mathrm{g}}\left(\varepsilon_{i j, k}^{\mathrm{p}}\right)
$$

with the elastic part defined by the standard Hookean response

$$
\psi_{\mathrm{e}}=\frac{1}{2} C_{i j k l} \varepsilon_{i j}^{\mathrm{e}} \varepsilon_{k l}^{\mathrm{e}}
$$

General forms of the local and nonlocal parts of the inelastic free energy, corresponding to power law hardening, are

$$
\begin{aligned}
\Psi_{\mathrm{p}} & =\frac{A \varepsilon_{0}^{-m}}{m+1}\left(\varepsilon_{i j}^{\mathrm{p}} \varepsilon_{i j}^{\mathrm{p}}\right)^{(m+1) / 2} \text { and } \\
\Psi_{\mathrm{g}} & =\frac{B \varepsilon_{0}^{-r} \ell^{r+1}}{r+1}\left(\varepsilon_{i j, k}^{\mathrm{p}} \varepsilon_{i j, k}^{\mathrm{p}}\right)^{(r+1) / 2}
\end{aligned}
$$

where $A$ and $B$ are hardening moduli, $m$ and $r$ are hardening exponents, $\varepsilon_{0}$ is a reference strain, and $\ell$ is the constitutive length scale. Evolution of plastic strain is further constrained by a dissipation potential

$$
g\left(\dot{\varepsilon}_{i j}^{\mathrm{p}}, \dot{\varepsilon}_{i j, k}^{\mathrm{p}}\right)=\sigma_{0} \sqrt{\dot{\varepsilon}_{i j}^{\mathrm{p}} \dot{\varepsilon}_{i j}^{\mathrm{p}}+\ell^{2} \dot{\varepsilon}_{i j, k}^{\mathrm{p}} \dot{\varepsilon}_{i j, k}^{\mathrm{p}}}
$$

such that in the absence of plastic strain gradients or in the large-scale limit $\ell=0$, it reduces to standard Mises plasticity.

The principle of minimum potential energy can be written as follows:

$$
E(\boldsymbol{u})=\int_{V}\left(\psi\left(\varepsilon_{i j}(\boldsymbol{u})-\varepsilon_{i j}^{\mathrm{p}}, \varepsilon_{i j}^{\mathrm{p}}, \varepsilon_{i j, k}^{\mathrm{p}}\right)-f_{i} u_{i}\right) \mathrm{d} V-\int_{S_{N}} t_{i} u_{i} \mathrm{~d} S \rightarrow \min
$$

where $u_{i}$ is the displacement field over the domain $V$ subject to boundary condition on the Dirichlet boundary $S_{D}, f_{i}$ are body forces, $t_{i}$ are the tractions on the Neumann boundary $S_{N}$, and $\varepsilon_{i j}(\boldsymbol{u})$ denotes the strain operator

$$
\varepsilon_{i j}(\boldsymbol{u})=\frac{u_{i, j}+u_{j, i}}{2}
$$


In addition, the rate of plastic straining is given by the minimum rate problem

$$
G\left(\dot{\varepsilon}_{i j}^{\mathrm{p}}\right)=\int_{V}\left(g\left(\dot{\varepsilon}_{i j}^{\mathrm{p}}, \dot{\varepsilon}_{i j, k}^{\mathrm{p}}\right)-\sigma_{i j} \dot{\varepsilon}_{i j}^{\mathrm{p}}\right) \mathrm{d} V \rightarrow \min
$$

Solutions that satisfy Eqs. (24) and (26) define the evolution of plastic strain in the solid.

Analytical solution to the problem defined by Eqs. (24) and (26) is possible if we resort to rigid-plastic and deformation theory approximations. Assuming elastic strain rates are negligible compared with plastic strain rates, we have

$$
\dot{\varepsilon}_{i j}^{\mathrm{p}} \approx \varepsilon_{i j}(\dot{\boldsymbol{u}})
$$

Under these conditions, Eq. (24) becomes

$E(\boldsymbol{u})=\int_{V}\left(\psi_{\mathrm{p}}\left(\varepsilon_{i j}(\boldsymbol{u})\right)+\psi_{\mathrm{g}}\left(\frac{\partial \varepsilon_{i j}(\boldsymbol{u})}{\partial x_{k}}\right)-f_{i} u_{i}\right) \mathrm{d} V-\int_{S_{N}} t_{i} u_{i} \mathrm{~d} S \rightarrow \min$

and Eq. (26) reduces to

$$
G(\dot{\boldsymbol{u}})=\int_{V}\left(g\left(\varepsilon_{i j}(\dot{\boldsymbol{u}}), \frac{\partial \varepsilon_{i j}(\dot{\boldsymbol{u}})}{\partial x_{k}}\right)+\frac{\mathrm{d} E(\boldsymbol{u})}{\mathrm{d} t}\right) \mathrm{d} V \rightarrow \min
$$

This formulation characterizes the evolution of the displacement field. By assuming proportional loading of the body, the deformation must also be proportional in time, which leads to the following minimum problem for the displacement

$$
\begin{aligned}
F(\boldsymbol{u})= & \int_{V}\left[g\left(\varepsilon_{i j}(\boldsymbol{u}), \frac{\partial \varepsilon_{i j}(\boldsymbol{u})}{\partial x_{k}}\right)+\psi_{\mathrm{p}}\left(\varepsilon_{i j}(\boldsymbol{u})\right)+\psi_{\mathrm{g}}\left(\frac{\partial \varepsilon_{i j}(\boldsymbol{u})}{\partial x_{k}}\right)\right] \mathrm{d} V \\
& -\int_{S_{N}} t_{i} u_{i} \mathrm{~d} S \rightarrow \min
\end{aligned}
$$

in the absence of body forces. It should be noted that the deformation theory energy functional that should be minimized in Eq. (30) is a combination of both free energy and dissipation.

3.2 Introduction of Fractional Gradients. As in our previous paper, we now extend the above theory to fractional derivatives of plastic strain. Assuming integrated fractional strain-gradient free energies on the form,

$$
\Psi_{\mathrm{g}}=\frac{B}{r+1} \frac{\ell^{s(r+1)}}{\varepsilon_{0}^{r+1}} \int_{V} \int_{V} \frac{\left|\varepsilon^{\mathrm{p}}\left(\boldsymbol{x}^{\prime}\right)-\varepsilon^{\mathrm{p}}\left(\boldsymbol{x}^{\prime \prime}\right)\right|^{r+1}}{\left|\boldsymbol{x}^{\prime}-\boldsymbol{x}^{\prime \prime}\right|^{d+s(r+1)}} \mathrm{d} V^{\prime} \mathrm{d} V^{\prime \prime}
$$

where $\boldsymbol{x}$ are the coordinates spanning the domain $V, d$ is the spatial dimension, and $s \in(0,1)$ is the fractional order of differentiation, and we write

$$
|\varepsilon|=\sqrt{\varepsilon_{i j} \varepsilon_{i j}}
$$

The fractional strain-gradient free energy functional in Eq. (31) becomes nonlocal by considering interactions at a distance instead of point-by-point evaluations of strain gradients in a constitutive law. The functional in Eq. (31) is known as the Gagliardo seminorm, see Ref. [32], and interpolates between the integrated forms of Eq. (22).

\section{Results}

The simple shear problem in Fig. 1 is solved using the approaches outlined earlier: SGP with energetic interfaces using an finite element method (FEM) solver, SGP using deformation theory, and FSGP again using deformation theory. The boundary value problem is one dimensional, and the only nonzero components of stress and plastic strain are denoted as $\tau$ and $\gamma^{p}$, respectively. It should be noted that, from the standard equilibrium Eq. (3), it can be concluded that $\tau$ is spatially constant at any instance during the deformation history.

4.1 Strain-Gradient Plasticity With Energetic Interface. Due to symmetry, only half of the problem in Fig. 1 is considered, from $y=0$ to $y=h$. At the interface/boundary, the higher order traction is denoted as $M$. Boundary conditions at the symmetry line are as follows:

$$
\text { At } y=0: \quad u_{x}=0, \quad \text { and } \quad \frac{\mathrm{d} \gamma^{\mathrm{p}}}{\mathrm{d} y}=0 \Rightarrow M=0
$$

The other boundary is occupied by the interface $\Gamma$. Boundary conditions are prescribed on side 2 to simulate the elastic material abutting the interface from the "outside" of the problem,

$$
\text { At } y=h: \quad u_{x}^{(2)}=u, \quad \text { and } \quad \gamma^{\mathrm{p}(2)}=0
$$

where $u$ is the prescribed displacement loading. The average total shear strain applied to the strip is then $\gamma=u / h$. As mentioned earlier, displacements are forced to be continuous across $\Gamma$ such that $u_{x}^{(1)}=u_{x}^{(2)}$, but the plastic strain $\gamma^{\mathrm{p}(1)}$ depends on the strength of the interface, $\kappa_{\Gamma}$.

The plastically deforming material is assumed to have material properties as follows: $E / \sigma_{0}=800$ is the ratio of Young's modulus to the initial large-scale yield stress in tension and Poisson's ratio $\nu=0.3$, which gives a ratio of the yield stress in shear $\tau_{0}=$ $\sigma_{0} / \sqrt{3}$ to the shear modulus $\mu=E /(2(1+\nu))$ as $\gamma_{0}=\tau_{0} / \mu=1.87 \times$ $10^{-3}$. Stress and strain results are normalized by $\tau_{0}$ and $\gamma_{0}$, respectively. The rate-independent limit is approached by setting the exponent $n=200$ and the numerical penalty factor $\eta=10^{-9}$, which only influences the solution to a very small extent in the "elastic region." Ideal local plasticity is assumed such that $\sigma_{\mathrm{f}}=\sigma_{0}$. The problem is solved with an in-house implicit finite element implementation for the SGP model [17], and a graded mesh (finer closer the interface) with 120 quadratic elements is used.

The effect of two parameter ratios are investigated: $h / \ell$, which compares the size of the plastically deforming region with the intrinsic length scale, and the ratio $\kappa_{\Gamma} /(\mu \ell)$, which governs the behavior of the interface.

In Fig. 2, the purple curves correspond to solutions with what is usually called micro hard boundary conditions, i.e., at the interface $\gamma^{p}=0$. As can be seen, the well-established size-dependent yield point is present, and halving the thickness of the strip roughly doubles the strengthening. A relative strengthening can here be defined as $\tau_{\mathrm{y}} / \tau_{0}-1$, where $\tau_{\mathrm{y}}$ is the stress where large plastic deformation occurs. This trend is explored in further detail below.

When the energetic interface is introduced, i.e., by letting $\kappa_{\Gamma}$ assume a finite value, the state at the boundary may be relaxed. The yielding initiates at the large-scale yield stress $\tau=\tau_{0}$, but the solution develops very differently from the local theory solution.

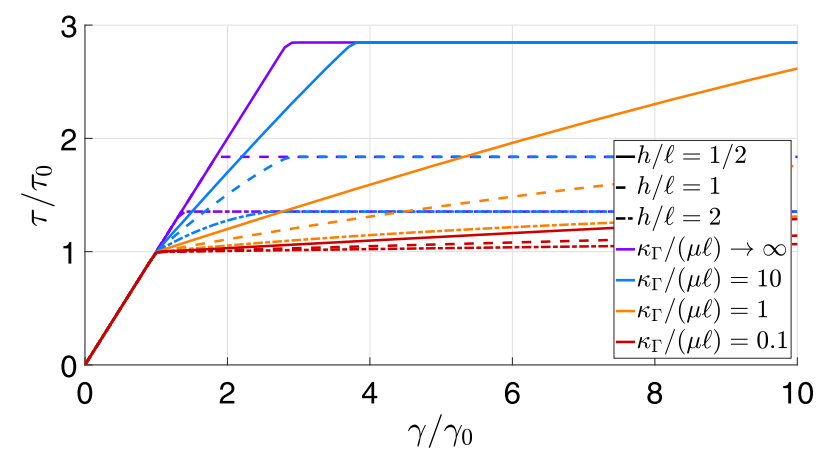

Fig. 2 Stress-strain response of the simple shear problem when different ratios of $h / \ell=[1 / 2,1,2]$ (indicated by the line style) and the ratio $\kappa_{\Gamma} /(\mu t)=[\infty, 10,1,0.1]$ (indicated by the line color) are considered (Color version online.) 
For large $\kappa_{\Gamma}$, the plastic strain evolution is severely reduced, and the stress-strain curve raises from the initial yield point with a slope approaching the elastic slope. After some small amount of plastic straining, it reaches the micro hard solution, and from there, the interface has played out its role and the solution proceeds according to the micro hard case. This can be seen in the case of $\kappa_{\Gamma} /(\mu \ell)=10$ in Fig. 2. At lower values of $\kappa_{\Gamma}$, the increase in shear stress with deformation proceeds with a lower slope, and as $\kappa_{\Gamma} \rightarrow 0$, the situation approaches the local theory solution.

In Figs. 3 and 4, plastic shear strain distributions are plotted to illustrate how the interface influences the solution. The plastic strain state is shown at three different average total strain levels $\gamma / \gamma_{0}=[2,3,4]$ and for the same four interface conditions as in Fig. 2 by the same color of the lines.

In Fig. 3, the size of the plastically deforming layer is $h / \ell=1 / 2$, corresponding to the case with the most pronounced size strengthening in Fig. 2. The weaker interfaces $\left(\kappa_{\Gamma} /(\mu \ell)=0.1\right.$ and 1) produce almost homogeneous plastic strain distributions, but the stronger interface, $\kappa_{\Gamma} /(\mu \ell)=1$, shows a reduced overall level of plastic strain compared with the weaker interface (which is close to the local plastic solution). The solution for $\kappa_{\Gamma} /(\mu \ell)=10$ is initially almost homogeneous, although with much reduced magnitude, but just below $\gamma / \gamma_{0}=4$, it becomes energetically favorable to develop strong gradients instead of additional plastic strain at the interface. Consequently, beyond this point the boundary behaves as a full constraint on plastic deformation locally.

In Fig. 4 , the size of the plastically deforming layer is $h / \ell=2$, corresponding to the case with the least pronounced size strengthening in Fig. 2. Here, the case $\kappa_{\Gamma} /(\mu \ell)=0.1$ behaves very close to the local theory solution. The solution for $\kappa_{\Gamma} /(\mu \ell)=1$ is less

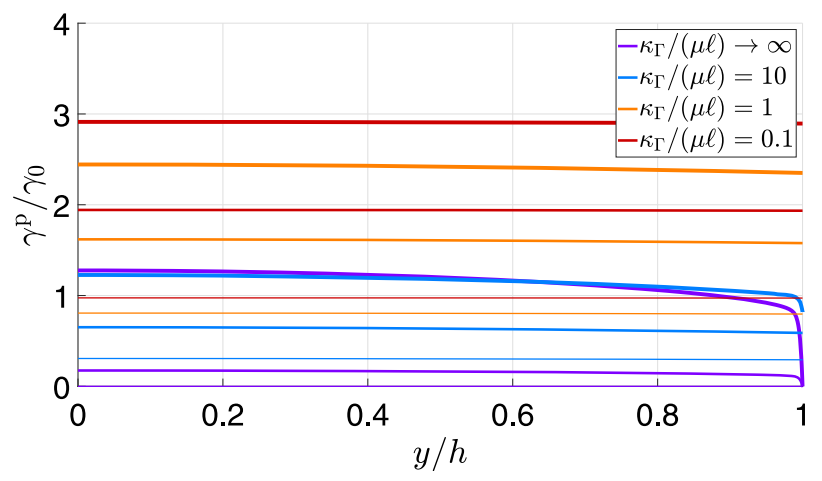

Fig. 3 Plastic shear strain distributions for layers of thickness $h / \ell=1 / 2$ at three different macroscopic shear strain levels $\gamma / \gamma_{0}=[2,3,4]$ (indicated by the increasing thickness of the lines and also by greater plastic strain levels)

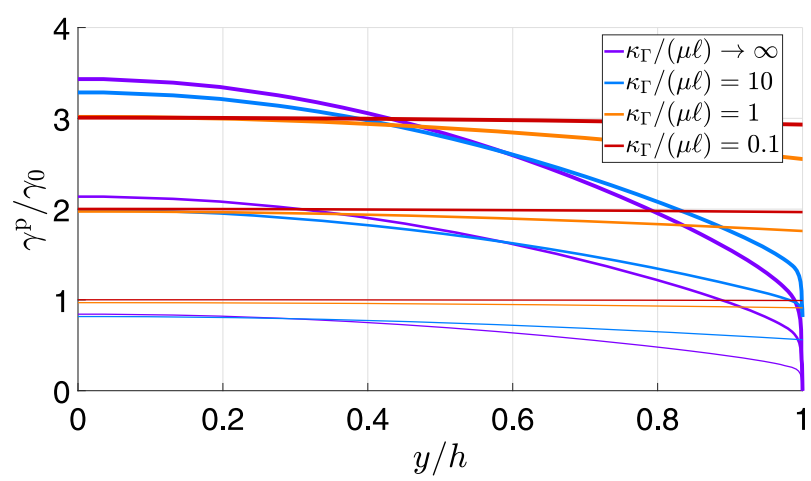

Fig. 4 Plastic shear strain distributions for layers of thickness $h / \ell=2$ at three different macroscopic shear strain levels $\gamma / \gamma_{0}=$ $[2,3,4]$ (indicated by the increasing thickness of the lines and also by greater plastic strain levels) homogeneous than in Fig. 3 but is closer to the local theory solution. The transition to micro hard when $\kappa_{\Gamma} /(\mu \ell)=10$ happens sooner than in Fig. 3, and both of the solutions with stronger interfaces show a clear departure from the local theory solution.

To analyze the scaling of the yield stress, an operative measure of yield stress $\tau_{\mathrm{y}}$ needs to be defined; not only because of the plastic strain compliance introduced by the interface but also because the bulk behavior is viscoplastic and, consequently, there inevitably is a small amount of plastic flow even at $\tau<\tau_{0}$. The average plastic shear strain is defined as follows:

$$
\left\langle\gamma^{\mathrm{p}}\right\rangle=\frac{1}{h} \int_{0}^{h} \gamma^{\mathrm{p}}(y) \mathrm{d} y
$$

and the yield stress is defined as the stress when $\left\langle\gamma^{\mathrm{p}}\right\rangle=\gamma_{\text {offset }}$. For the purpose of this investigation, the actual value of $\gamma_{\text {offset }}$ does not turn out to be of much importance as long as $0.2<\gamma_{\text {offset }} /$ $\gamma_{0}<2$, approximately. A value of $\gamma_{\text {offset }}=0.5 \gamma_{0}$ have been used here.

In Fig. 5, FEM results are plotted, as circles, for the relative yield stress strengthening $\tau_{\mathrm{y}} / \tau_{0}-1$ against the normalized material thickness $h / \ell$. The different colors of the circles correspond to different interface strengths according to the legend. The results span across two decades in both thickness and interface strength. The purpose of this investigation is to investigate the linear scaling with the inverse of thickness and whether it changes upon relaxation of the boundary conditions. To that end, a function on the form

$$
\tau_{\mathrm{y}}=\tau_{0}+k \frac{\ell}{h}
$$

where $k$ is the only parameter, has been fitted to each set of SGP results. The functions were made to match exactly at $h / \ell=0.2$ and are plotted in Fig. 5 as solid lines of corresponding color. It can be seen that in the regime $h / \ell<1$, the numerical results follow a linear scaling relationship to a good approximation, independent of the interface strength. The magnitude of the strengthening $k$ depends on the value of $\kappa_{\Gamma}$. For example, $k \rightarrow \tau_{0}$ as $\kappa_{\Gamma} \rightarrow \infty$ when $h / \ell \ll 1$. This is not fully developed in the cases shown in Fig. 2 and $k \approx 0.85 \tau_{0}$ there. In the regime $h / \ell>1$, the SGP results departs from the linear trend as the slope increases, implying an even stronger scaling than $1 / h$ in this regime.

4.2 Deformation Theory Strain-Gradient Plasticity Results. The problem under consideration is the simple shear problem defined above but without the additional interface description, i.e., micro hard conditions prevail at $y= \pm h$. It was shown in Ref. [21] that the deformation theory formulation of SGP (Sec. 3.1) gives a length scale-dependent yield stress in shear,

$$
\tau_{\mathrm{y}}=\tau_{0}\left[1+\frac{\ell}{h}\right]
$$

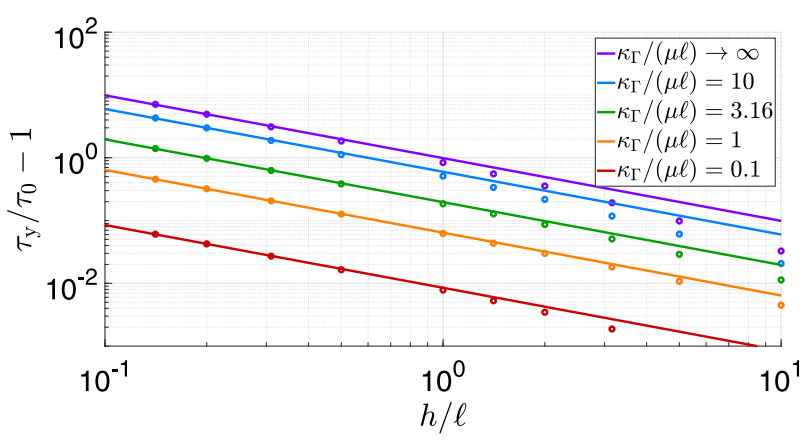

Fig. 5 Size-dependent strengthening shows a clear linear trend in the regime of strong strengthening independent of the strength of the interface. Circles indicate SGP predictions of strengthening from FEM solutions. Solid lines are fitted curves on the form $\tau_{y}=\tau_{0}+k(\ell / h)$. (Color version online.) 
when the inelastic free energy contributions in Eq. (22) are linear growth functions, i.e., $m=r=0$. Specifically, for a size-dependent yield stress to be predicted, the nonlocal energy $\psi_{\mathrm{g}}$ has to be a linear function of the gradients of plastic strain. This shows that this simplified deformation theory approach reproduces results from Sec. 4.1 above and other similar formulations (e.g., Ref. [33]). For superlinear growth of the nonlocal energy, the size dependence of the yield point vanishes, again in accordance with what is usually termed energetic contributions in conventional SGP, see, e.g., Ref. [34].

4.3 Deformation Theory Fractional Strain-Gradient Plasticity Results. For an infinite solid containing a layer of thickness $2 h$ deformed in simple shear and otherwise undeformed (i.e., the simple shear configuration under consideration), the free energy in Eq. (31) can be written as follows:

$$
\Psi_{\mathrm{g}}=\frac{B}{r+1} \frac{\ell^{s(r+1)}}{\gamma_{0}^{r+1}} \int_{-\infty}^{\infty} \int_{-\infty}^{\infty} \frac{\left|\gamma^{\mathrm{p}}\left(y^{\prime}\right)-\gamma^{\mathrm{p}}\left(y^{\prime \prime}\right)\right|^{r+1}}{\left|y^{\prime}-y^{\prime \prime}\right|^{1+s(r+1)}} \mathrm{d} y^{\prime} \mathrm{d} y^{\prime \prime}
$$

with any necessary redefinition of the modulus $B$. The minimization procedure with a linear growth nonlocal energy $(r=0)$ and ideal local plasticity $\left(m=0\right.$, and $\left.A=\tau_{0} \gamma_{0}\right)$ results in a condition for nonzero average (plastic) shear strain $\langle\gamma\rangle \neq 0$ if

$$
\tau=\frac{h+\ell^{s} h^{1-s}}{h} \tau_{0}
$$

which identifies the yield stress in shear as follows:

$$
\tau_{\mathrm{y}}=\tau_{0}\left[1+\left(\frac{\ell}{h}\right)^{s}\right]
$$

The result in Eq. (40) is similar to Eqs. (36) and (37) but with the difference that the fractional order of differentiation, $s$, comes out as the scaling exponent. If $s \rightarrow 1$, the situation returns to the previously considered $1 / h$ scaling laws from conventional SGP. This is expected since as $s \rightarrow 1$ the fractional differentiation becomes the classic derivative and the free energy contribution $\Psi_{\mathrm{g}}$ depends on the first derivative of the plastic strain. In other words, the model reproduces the case when the free energies in Eq. (22) are considered.

\section{Summary and Concluding Remarks}

In a previous paper [21], we have developed a theory of FSGP and applied it to the simple shear problem. In Ref. [21], we have also shown that the theory can be made to fit exactly the experimentally observed trends [1-3]. Therefore, FSGP is a candidate model to solve the size-scaling discrepancy between experiments and conventional SGP models noted by Ref. [10].

However, the precise nature of the plastic strain state at the boundary of a small and confined volume is difficult to ascertain from available experiments, which raises the issue of whether higher order boundary conditions other than micro hard, in conjunction with conventional SGP, result in scalings that more closely match experiments. In this paper, we have shown that a general class of relaxed boundary conditions in the form of an energetic interface, which effectively interpolates between the two extreme (micro hard and micro free) boundary conditions, does not relax the size scaling of conventional SGP. The relaxed boundary conditions reduce the magnitude of the size-dependent strengthening with the result that larger strengthening effects are pushed further and further away from relevant size scales when compared with a fixed length scale parameter. Therefore, if the analysis is not done properly and results from numerics are fitted to a $h^{-\alpha}$ power law, it may erroneously appear that the scaling is different from $\alpha=1$, since the SGP effects may not be fully developed in the length interval considered. Such a model can of course reproduce the results that it is fitted to, but any predictions at a smaller size range necessarily overestimate the yield stress relative to experiments.
Therefore, we conclude that both conventional SGP and SGP with boundary conditions relaxed through an energetic interface result in a yield stress that scales linearly with the inverse of layer thickness in the simple shear configuration. This scaling is grossly at odds with observational evidence [1-3]. By contrast, the FSGP model can be made to match the data by identifying the order of differentiation with the observed scaling exponent.

It bears emphasis that Ref. [29] also focuses on boundary conditions of conventional SGP and how they influence the scaling. Their findings vis a vis scaling are in sharp contrast to the energetic-interfaces analysis of Sec. 4.1. Remarkably, a careful reexamination reveals that the boundary conditions proposed by Ref. [29] are in fact reverse relative to the conditions experienced by the boundary in the energetic interface model. Thus, in the model described in Ref. [29], the plastic strain is at first constrained to vanish and, following the attainment of a critical value, the plastic strain gradient is constrained to be constant. Energetic interfaces also behave as evolving boundary conditions. Thus, at the initiation of the plastic flow, the behavior can best be described as a Robintype condition, namely, a weighted combination of a Dirichlet and a Neumann boundary conditions. If the parameter $\kappa_{\Gamma} /(\mu \ell)$ is small, the constraint on plastic flow is negligible and, consequently, the development of a gradient is restricted. Conversely, if $\kappa_{\Gamma} /(\mu \ell)$ is large, the plastic flow is reduced compared with the bulk and a strong gradient develops. At some later point in the load history, the interface transitions to a delayed micro-hard condition (compared with Ref. [17] for details) and no further plastic strain accumulates. However, since the bulk material continues to accumulate plastic strain the gradient at the interface increases. This model comparison suggests that the precise sequence in which higher order boundary conditions are applied in conventional SGP strongly influences the outcome.

\section{Acknowledgment}

C.F.O.D. gratefully acknowledges financial support from Vetenskapsrådet (VR E0566901) and the VINN Excellence Center Hero-m funded by Vinnova. M.O. is grateful for the support of the Deutsche Forschungsgemeinschaft through the Sonderforschungsbereich 1060 "The mathematics of emergent effects" at the Hausdorff Center for Mathematics of the University of Bonn, Germany.

\section{References}

[1] Mu, Y., Chen, K., and Meng, W. J., 2014, "Thickness Dependence of Flow Stress of $\mathrm{Cu}$ Thin Films in Confined Shear Plastic Flow," MRS Commun. Res. Lett., 4(3), pp. 126-133.

[2] Mu, Y., Zhang, X., Hutchinson, J. W., and Meng, W. J., 2016, "Dependence of Confined Plastic Flow of Polycrystalline $\mathrm{Cu}$ Thin Films on Microstructure," MRS Commun. Res. Lett., 6(3), pp. 289-294.

[3] Mu, Y., Zhang, X., Hutchinson, J. W., and Meng, W. J., 2017, "Measuring Critical Stress for Shear Failure of Interfacial Regions in Coating/Interlayer/ Substrate Systems Through a Micro-Pillar Testing Protocol," J. Mater. Res., 32(8), pp. 1421-1431

[4] Hall, E. O., 1951, "The Deformation and Ageing of Mild Steel: III Discussion of Results," Proc. Phys. Soc. Section B, 64(9), pp. 747-753.

[5] Petch, N. J., 1953, "The Cleavage Strength of Polycrystals," J. Iron Steel Inst., 174, pp. 25-28.

[6] Fleck, N. A., and Hutchinson, J. W., 2001, "A Reformulation of Strain Gradient Plasticity,” J. Mech. Phys. Solids., 49(10), pp. 2245-2271.

[7] Gudmundson, P., 2004, "A Unified Treatment of Strain Gradient Plasticity," J. Mech. Phys. Solids., 52(6), pp. 1379-1406

[8] Fleck, N. A., and Willis, J. R., 2009, "A Mathematical Basis for Strain-Gradient Plasticity Theory—Part I: Scalar Plastic Multiplier,” J. Mech. Phys. Solids., 57(1), pp. 161-177.

[9] Fleck, N. A., and Willis, J. R., 2009, "A Mathematical Basis for Strain-Gradient Plasticity Theory. Part II: Tensorial Plastic Multiplier," J. Mech. Phys. Solids., 57(7), pp. 1045-1057

[10] Evans, A. G., and Hutchinson, J. W., 2009, "A Critical Assessment of Theories of Strain Gradient Plasticity," Acta. Mater., 57(5), pp. 1675-1688.

[11] Stölken, J. S., and Evans, A. G., 1998, “A Microbend Test Method for Measuring the Plasticity Length Scale,” Acta. Mater., 46(14), pp. 5109-5115. 
[12] Ehrler, B., Hou, X., P’Ng, K. M. Y., Walker, C. J., Bushby, A., and Dunstan, D. J., 2008, "Grain Size and Sample Size Interact to Determine Strength in a Soft Metal," Philos. Mag., 88(25), pp. 3043-3050.

[13] Idiart, M. I., Deshpande, V. S., Fleck, N. A., and Willis, J. R., 2009, "Size Effects in the Bending of Thin Foils," Int. J. Eng. Sci., 47(11-12), pp. 1251-1264.

[14] Fleck, N. A., and Hutchinson, J. W., 1997, "Strain Gradient Plasticity," Advances in Applied Mechanics, Vol. 33, J. W. Hutchinson, and T. Y. Wu, eds., Elsevier, New York, pp. 295-361.

[15] Idiart, M. I., and Fleck, N. A., 2009, "Size Effects in the Torsion of Thin Metal Wires," Modell. Simul. Mater. Sci. Eng., 18, pp. 1-10.

[16] Niordson, C. F., and Hutchinson, J. W., 2011, "Basic Strain Gradient Plasticity Theories With Application to Constrained Film Deformation," J. Mech. Mater. Struct., 6(1-4), pp. 395-416.

[17] Dahlberg, C. F. O., and Faleskog, J., 2013, “An Improved Strain Gradient Plasticity Formulation With Energetic Interfaces: Theory and a Fully Implicit Finite Element Formulation," Comput. Mech., 51(5), pp. 641-659.

[18] Abu Al-Rub, R. K., and Voyiadjis, G. Z., 2006, "A Physically Based Gradient Plasticity Theory,” Int. J. Plast., 22(4), pp. 654-684.

[19] Dahlberg, C. F. O., and Boåsen, M., 2019, "Evolution of the Length Scale in Strain Gradient Plasticity," Int. J. Plast., 112, pp. 220-241.

[20] Scherer, J. M., Besson, J., Forest, S., Hure, J., and Tanguy, B., 2019, "Strain Gradient Crystal Plasticity With Evolving Length Scale: Application to Voided Irradiated Materials," Euro. J. Mech. A/Solids, 77, p. 103768.

[21] Dahlberg, C. F. O., and Ortiz, M., 2019, "Fractional Strain-Gradient Plasticity," Euro. J. Mech. A/Solids, 75, pp. 348-354.

[22] Tarasov, V. E., and Aifantis, E. C., 2019, "On Fractional and Fractal Formulations of Gradient Linear and Nonlinear Elasticity," Acta Mechanica, 230(6), pp. 2043-2070.
[23] Lakes, R. S., 1998, Viscoelastic Solids, 1st ed., CRC Press, Boca Raton, FL.

[24] Sun, Y., and Zheng, C., 2019, "Fractional-Order Modelling of State-Dependent Non-Associated Behaviour of Soil Without Using State Variable and Plastic Potential," Adv. Differ. Equ., 2019, p. 103768.

[25] Sumelka, W., 2018, "On a General Numerical Scheme for the Fractional Plastic Flow Rule,” Mech. Mater., 116, pp. 120-129.

[26] Heyden, S., Li, B., Weinberg, K., Conti, S., and Ortiz, M., 2015, "A Micromechanical Damage and Fracture Model for Polymers Based on Fractional Strain-Gradient Elasticity,” J. Mech. Phys. Solids, 74, pp. 175-195.

[27] Heyden, S., Conti, S., and Ortiz, M., 2015, "A Nonlocal Model of Fracture by Crazing in Polymers," Mech. Mater., 90, pp. 131-139.

[28] Conti, M., and Ortiz, M., 2016, "Optimal Scaling in Solids Undergoing Ductile Fracture by Crazing," Arch. Rational Mech. Anal., 219(2), pp. 607-636.

[29] Kuroda, M., and Needleman, A., 2019, "A Simple Model for Size Effects in Constrained Shear," Extreme Mech. Lett., 33, p. 100581.

[30] Fredriksson, P., and Gudmundson, P., 2005, "Size-Dependent Yield Strength of Thin Films," Int. J. Plast., 21(9), pp. 1834-1854.

[31] Dahlberg, C. F. O., Faleskog, J., Niordson, C. F., and Legarth, B. N, 2013, "A Deformation Mechanism Map for Polycrystals Modeled Using Strain Gradient Plasticity and Interfaces That Slide and Separate," Int. J. Plasticity, 43, pp. 177-195.

[32] Di Nezza, E., Palatucci, G., and Valdinoci, E., 2012, "Hitchhiker's Guide to the Fractional Sobolev Spaces,” Bull. Des. Sci. Math., 136(5), pp. 521-573.

[33] Gudmundson, P., and Dahlberg, C. F. O., 2019, "Isotropic Strain Gradient Plasticity Model Based on Self-Energies of Dislocations and the Taylor Model for Plastic Dissipation,” Int. J. Plast., 121, pp. 1-20.

[34] Niordson, C. F., and Legarth, B. N., 2010, "Strain Gradient Effects on Cyclic Plasticity,” J. Mech. Phys. Solids., 58(4), pp. 542-557. 